\section{Low HDL despite statin-lowered LDL cholesterol levels raises risk of cardiovascular events}

Low levels of HDL and high levels of LDL cholesterol are both important predictors of cardiovascular disease. LDL cholesterol levels are effectively lowered by statin therapy, significantly decreasing the risk of cardiovascular events. The impact of HDL cholesterol levels in patients with very low LDL cholesterol has not, however, been investigated.

Barter et al. studied the predictive value of HDL cholesterol levels as part of the Treating to New Targets (TNT) study. They followed up 9,770 patients who were being actively treated with statins. The main outcome measure was the time to first major cardiovascular event. This end point included fatal or nonfatal stroke, nonfatal myocardial infarction, resuscitation after cardiac arrest, and death from coronary heart disease.

Statin-treated patients who achieved the lowest levels of LDL cholesterol-less than $1.81 \mathrm{mmol} / \mathrm{l}(<70 \mathrm{mg} / \mathrm{dl})$-were still likely to suffer an adverse cardiovascular event if they also had low HDL cholesterol concentrations. Patients with low LDL and high HDL cholesterol levels were at the lowest risk. The concentration of HDL cholesterol remained a significant predictor of major cardiovascular events after all other baseline risk factors, including baseline LDL cholesterol level, were taken into account.

The authors conclude that HDL cholesterol levels are still predictive of major cardiovascular events in patients treated with statins, even in those who achieve the target LDL cholesterol level of less than $1.81 \mathrm{mmol} / \mathrm{l}(70 \mathrm{mg} / \mathrm{dl})$.

Original article Barter P et al. (2007) HDL cholesterol, very low levels of LDL cholesterol, and cardiovascular events. N Engl J Med 357: 1301-1310

\section{ICDs for heart failure are more commonly used among white men}

Implantable cardioverter-defibrillators (ICDs) are recommended for heart failure patients with a left ventricular ejection fraction of $30 \%$ or lower. Hernandez and colleagues reviewed data from the heart failure module of the AHA's Get With the Guidelines Program, in order to assess the use of ICD therapy among eligible patients.
The final cohort comprised 13,034 patients from 217 hospitals.

The frequency of ICD use was low-only 4,615 (35.4\%) hospitalized patients who were eligible for a device had received, or were scheduled to receive, an ICD at discharge. Significant disparities in the use of ICD therapy were seen between subgroups, with men more likely to receive a device than women, and white patients more likely to undergo device implantation than black patients. The frequencies of ICD use in eligible patients were $43.6 \%$ for white men, $33.4 \%$ for black men, $29.8 \%$ for white women and $28.2 \%$ for black women. These discrepancies remained after adjusting for a range of patient and hospital factors-compared with white men, the adjusted odds ratios for receiving an ICD were 0.73 for black men $(95 \% \mathrm{Cl}$ 0.60-0.88; $P=0.001$ ), 0.62 for white women $(95 \% \mathrm{Cl} 0.56-0.68 ; P<0.001)$ and 0.56 for black women (95\% Cl 0.44-0.71; $P<0.001$ ).

The findings of this study support the introduction of programs to promote the use of ICDs in eligible heart failure patients. Further study is required to understand the reasons for the disparities that currently exist in the use of this therapy.

Original article Hernandez AF et al. (2007) Sex and racial differences in the use of implantable cardioverter-defibrillators among patients hospitalized with heart failure. JAMA 298: $1525-1532$

\section{PFO closure without a permanent implant is safe and feasible}

Percutaneous closure of a patent foramen ovale (PFO) typically involves the implantation of a permanent closure device, but this can lead to long-term complications such as thrombus formation. Sievert et al. reported the results from the first human study to assess the PFx ${ }^{\circledR}$ closure system (Cierra, Inc., Redwood City, CA). The system allows the operator to approach via the right atrium and seal the PFO using radiofrequency energy, without the need for implantation of a permanent device. In total, 30 patients (mean age 48 years) took part in the study, all of whom had a PFO and a history of transient ischemic attack, cryptogenic stroke or paradoxical embolism.

Sievert and colleagues' findings indicate that the PFx is safe-no serious adverse events related to the procedure were documented 